\title{
¿Se puede motivar a nuestros alumnos para que cursen - con agrado e interés- nuestras asignaturas de Matemáticas?
}

\author{
Roque Molina Legaz \\ Dpto Matemática Aplicada y Estadística \\ Universidad Politécnica de Cartagena \\ Antiguo Hospital de Marina, 30202 - Cartagena \\ e-mail: roque.molina@upct.es \\ Presentado en eXIDO18 (2018)

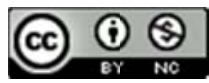

\section{RESUMEN}

En esta charla se trata de reflexionar (e incitar al debate con el resto de asistentes a estas Jornadas Exido 2018) sobre los problemas con los que habitualmente nos encontramos los docentes a la hora de motivar a nuestros alumnos para que comprendan la utilidad que tienen las asignaturas de Matemáticas a cursar en su grado, y que, en la medida de lo posible, entiendan que se puede disfrutar con las mismas, así como que vean a estas asignaturas como parte de un todo, en lugar de como algo aislado en su formación y que no tienen más remedio que superar para obtener su ansiado grado.

Palabras clave: motivación, aprendizaje, docencia, Matemáticas.

\section{INTRODUCCIÓN}

Desde la experiencia de los ya más de 30 años que hemos dedicado a impartir asignaturas de Matemáticas en estudios de Ingeniería (especialmente en las relacionadas con Ingeniería Industrial), se trata de reflexionar sobre técnicas motivacionales, así como sobre la carencia que de las mismas tienen nuestros alumnos a la hora de abordar sus asignaturas de Matemáticas. Se trata de compartir, a través de una charla distendida en el marco de las Jornadas Exido 2018, algunas reflexiones sobre este tema, y poder plantear a todos los asistentes si se considera que el problema objeto del título de esta charla tiene solución y cual (o cuales) son las técnicas más eficientes para responder a la cuestión propuesta.

La cuestión principal a contestar podría ser: ¿Se puede motivar a nuestros alumnos para que cursen con agrado e interés nuestras asignaturas de Matemáticas... aparte de para conseguir su tan ansiado aprobado en las mismas?

De antemano solicitamos -y casi suplicamos- la comprensión de los asistentes -y de futuros posibles lectores-, porque seguro que no vamos a ser muy originales en esta exposición, ni somos muy optimistas en cuanto al resultado de la pregunta planteada. Insistimos que la intención principal es la de 
reflexionar sobre el título de la charla, analizando fortalezas y debilidades de lo realizado en clase cuando se imparten nuestras asignaturas y, en base a estas consideraciones, debatir sobre este tema, y, por qué no, absorber (por no decir "copiar descaradamente") cualquier sugerencia que, en el debate posterior, se pueda dar al respecto en base a la experiencia de los asistentes a esta charla.

$\mathrm{Si}$-con falsa modestia- nos podemos considerar buenos docentes, que somos vehementes en nuestras explicaciones, que hilamos bien el discurso a la hora de explicar los conceptos y sus aplicaciones, que estamos suficientemente motivados y nos creemos lo que contamos en nuestras asignaturas... entonces, ¿por qué no somos capaces de comunicar correctamente? ¿por qué nuestros alumnos -y lo que es más grave, muchos de los compañeros profesores de otras áreas- consideran que nuestras asignaturas de Matemáticas son una "isla" separada del continente que forman casi todas las demás asignaturas de su título? ¿Por qué hay alumnos en clase más pendientes de sus teléfonos móviles o de sus tabletas y ordenadores que de las explicaciones que les estamos dando?

Como ya se ha comentado, este artículo va especialmente referenciado a experiencias en asignaturas de Matemáticas en titulaciones de Ingeniería Industrial, dejando constancia de que entendemos que igual la experiencia puede ser un poco distinta -o quizás no- para los docentes de asignaturas del grado de Matemáticas: la discusión puede ser diferente para unas que para otras asignaturas, si bien no a la hora de motivar a los alumnos, sí a la hora de conseguir la misma a través de la "utilidad" de nuestras asignaturas. Y es que la diferencia no es baladí, ya que en Ingeniería las asignaturas de Matemáticas (encuadradas en su mayoría como contenidos básicos) no dejan de ser una herramienta de apoyo, más o menos necesaria, en cuanto a la formación de los alumnos, mientras que en el grado de Matemáticas sí que se pueden considerar un fin en sí mismo. Seguro que también habrá oyentes y lectores discrepantes -y con toda la razón- en lo afirmado en este último párrafo.

\section{REFLEXIONES}

¿Por qué entendemos que es necesaria e imprescindible la motivación en nuestras asignaturas de Matemáticas? Además de porque la mayoría de alumnos carecen de ella, porque consideramos que básicamente se verifica la siguiente equivalencia (que no es exclusiva de asignaturas de Matemáticas):

\section{MOTIVACIÓN $\Leftrightarrow$ APRENDIZAJE}

Evidentemente un alumno motivado (en nuestras disciplinas) será un alumno más receptivo para aprender; y recíprocamente, un alumno que aprende y que entiende lo que se le explica y que comprende la utilidad de lo aprendido, será un alumno motivado. $\mathrm{Y}$ sin olvidar otra de las máximas relacionadas con aprendizaje en el aula: si el alumno se encuentra motivado para el estudio de cualquier disciplina, bien seguro que en el momento en que sea titulado y abandone las aulas, seguirá aprendiendo el resto de su vida. Posiblemente éste sea uno de los aspectos a los que no se le presta la suficiente atención, y 
que consideramos que debe de ser una de las principales obligaciones del sistema universitario.

No obstante, nuestros alumnos suelen ser más prácticos, y la mayoría de ellos suelen ver la anterior equivalencia en la forma

$$
\text { MOTIVACIÓN } \Leftrightarrow \text { ÉXITO }
$$

lo que en realidad equivale, traduciéndolo a su lenguaje, a

$$
\text { MOTIVACIÓN } \Leftrightarrow \text { APROBADO }
$$

Es de resaltar la escasa existencia de artículos sobre motivación para estudiar asignaturas Matemáticas dirigidos a estudiantes universitarios; por el contrario, sí que existe una gran variedad de los mismos para intentar motivar -en Matemáticas y en otras disciplinas- en las etapas educativas anteriores. En los mismos suelen abundar las experiencias prácticas; con posterioridad se verá si es posible adaptar algunas de las mismas a las asignaturas de Matemáticas en la universidad.

Si por ejemplo en Google buscamos "motivación+Matemáticas+universidad” es posible encontrarse artículos como los que se incluyen en el siguiente pantallazo:

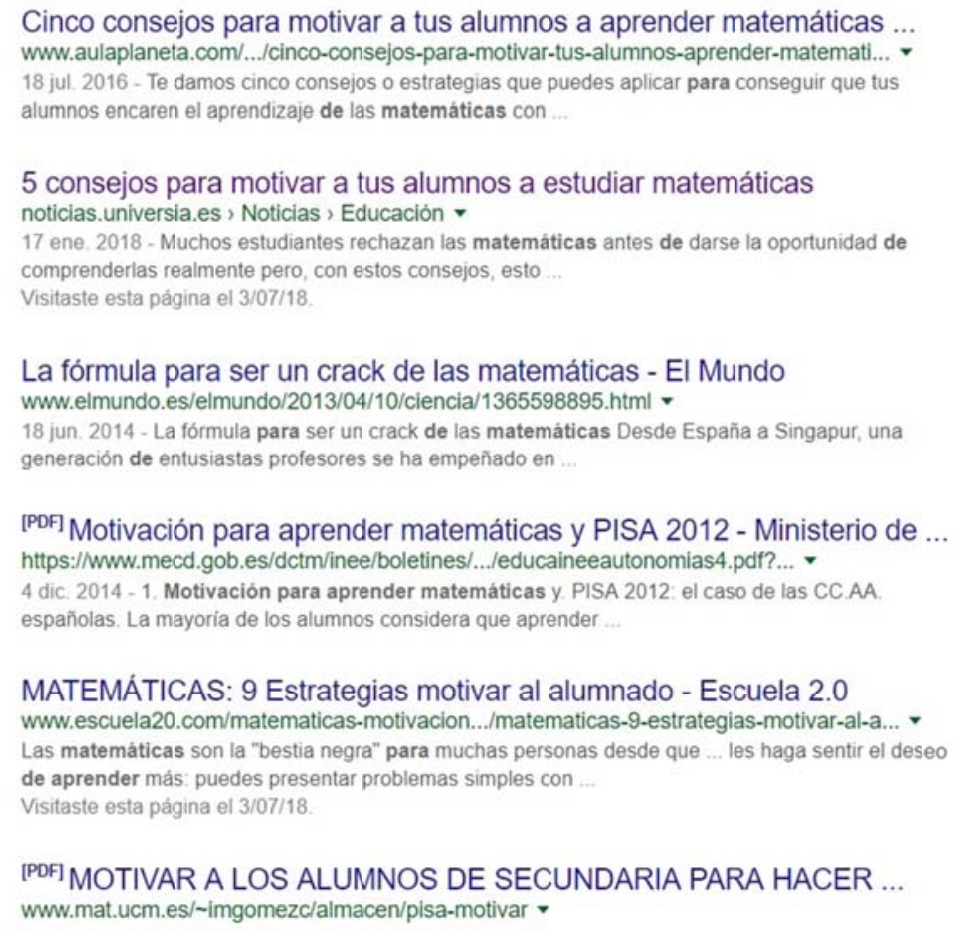

aunque siempre van dirigidos a estudiantes de primaria y/o secundaria.

Otro aspecto a considerar es el de la "mala prensa" que siempre han tenido nuestras asignaturas, posiblemente por la dificultad intrínseca que al parecer 
conllevan -aunque bien seguro que esta dificultad no es mayor que en otras disciplinas. Así, podemos encontrarnos artículos como
:三 MENú
EL. ESPAÑOL

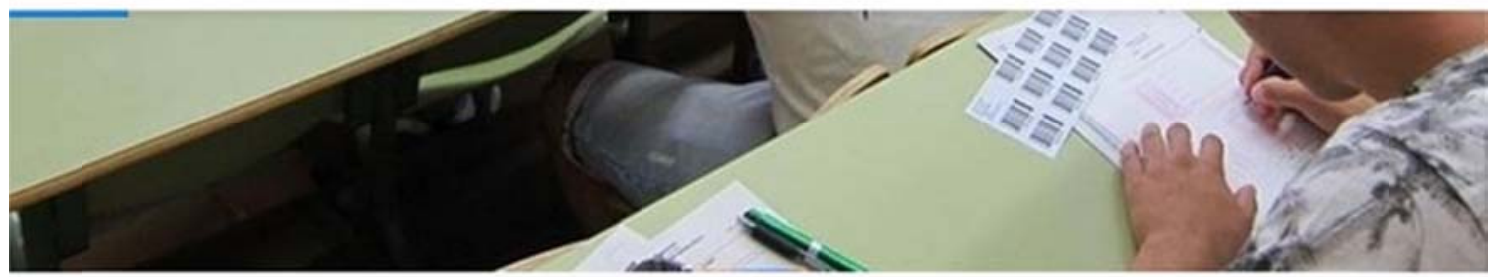

SOCIEDAD

\section{Dime qué carrera estudias y te diré cuántas asignaturas suspenderás}

Ingenierias, Física, Matemáticas y Estadistica son los estudios donde los universitarios suspenden más créditos.

30 diciembre, 2016 - 02:44

y alguno mucho más demoledores, como el siguiente

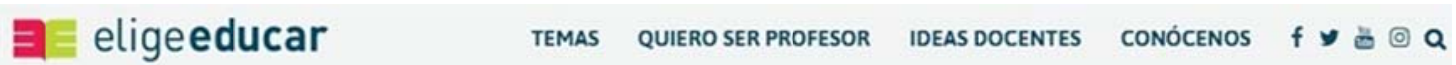

\section{“El $80 \%$ de lo que se aprende en la asignatura de matemática no sirve para nada"}

\section{Conrad Wolfram, físico que está cambiando la forma de enseñar matemáticas en Estonia, apuesta por eliminar el cálculo a mano}

Posiblemente esta mala fama haya contribuido a un descenso de alumnos matriculados en nuestras escuelas de ingeniería; aunque seguro que también han influido otros factores externos, como el trato que actualmente se da a las asignaturas tecnológicas en la ESO y en bachillerato.

\section{PLANTEAMIENTO DEL PROBLEMA}

Normalmente, cuando se teoriza sobre el término motivación, y entre sus tipos y clasificaciones, se suele destacar aquella que la clasifica en motivación intrínseca (la que se tiene por la mera satisfacción de adquirir nuevos conocimientos) y la motivación extrínseca (que va ligada a obtener determinadas ventajas; por ejemplo, aprobar la asignatura por ser necesaria para la obtención del título). Qué duda cabe que lo ideal sería lograr la motivación intrínseca, aunque pocos alumnos -refiriéndonos a Matemáticasmanifiestan la misma a no ser que previamente se les motive extrínsecamente. Es el profesor el que debe de actuar para inculcarles el entusiasmo por 
aprender, por lo que es imprescindible la actitud del mismo. Para ello, consideramos que el mismo debe de:

- Ser capaz de manifestar su motivación a sus alumnos, transmitiendo su entusiasmo por la materia y contagiarles el mismo.

- Hay que saber motivarles desde el primer instante, para lo que los alumnos deben conocer desde el inicio los objetivos a conseguir, que deben de tener una actitud positiva para lograrlos, y que ellos son los actores principales en la tarea a realizar.

- Conseguir que la motivación se alargue a lo largo del tiempo, para que así los alumnos deseen seguir aprendiendo una vez terminados sus estudios.

Sobre el papel, éstas parecen acciones muy concretas, pero es evidente que chocamos con una realidad tangible:

- Que se trata de asignaturas de Matemáticas.

- Que tenemos un montón de contenidos a impartir en nuestras asignaturas en las 15 semanas disponibles por cuatrimestre para cada asignatura.

- Que los alumnos tienen 4 o 5 asignaturas más en cada cuatrimestre, y que directa o indirectamente compiten con las nuestras.

- La creencia de que nuestras asignaturas son poco menos que el centro del universo, y que son imprescindibles sus contenidos para ser un buen ingeniero.

- Y con el tipo de evaluación que ahora llevamos (trabajos puntuables, controles, prácticas,...) mal se le tiene que dar a los alumnos para no obtener una nota próxima al aprobado, lo que igual les puede llevar a superar con relativo éxito la asignatura sin tener en absoluto que esforzarse ni motivarse.

$Y$ todo esto sin que entremos a valorar la formación inicial que portan los alumnos que llegan a nosotros, ni su grado de madurez, ni su motivación para estudiar estas titulaciones, ni muchos otros factores externos que traen en su "mochila" cuando acceden a la universidad. Y sin embargo, al parecer sí que han estado motivados en bachillerato, al tener como objetivo -muchos de ellos- conseguir la mayor nota posible en los exámenes de acceso a la universidad. Aunque no es este último un ejemplo ilustrativo del tipo de motivación -evidentemente extrínseca- que nos ha llevado a realizar las reflexiones de esta charla; sin embargo, creemos que sí que pone de manifiesto un término al que nos vamos a referir con posterioridad: egoísmo bien entendido; el alumno se mueve "por interés"- y que consideramos que se puede aprovechar en nuestro favor.

Antes de intentar dar respuesta al interrogante del título, creemos necesario que se reflexione sobre algunos interrogantes del por qué se ha llegado hasta la actual situación. Así, creemos que hemos de plantearnos cuestiones como las siguientes:

- ¿ ¿Por qué hay que enseñar Matemáticas en Ingeniería? 
- ¿Qué Matemáticas hay que enseñar? ¿Cómo se deben de enseñar?

- ¿Qué es lo que los profesores de Matemáticas hacemos en nuestras asignaturas y que es lo que de nosotros se espera que hagamos? Hay que tener en cuenta que estas asignaturas, al menos en lo que a estudios de Ingeniería y similares afecta, no son "finalistas" sino que son "medio" para la comprensión de las restantes (y también para un futuro desempeño del titulado).

- ¿Cómo ven estas asignaturas nuestros alumnos? Alumnos que, por ejemplo, lo que quieren es ser ingenieros, no matemáticos

En relación a estas cuestiones, creemos que estamos más o menos de acuerdo -los profesores- en el porqué hay que explicar Matemáticas en Ingeniería, y también en cuanto a los contenidos a impartir de las mismas, pero igual no se tiene tan claro el cómo impartirlas y, lo que más puede ser preocupante, no tenemos ni idea de cómo los alumnos ven nuestras asignaturas.

Igual, si nos centramos en responder esta última cuestión, se tendrá parte del problema -de la motivación- solucionado.

Se podría comenzar por intentar dar respuesta al siguiente interrogante: ¿Por qué los alumnos ven las Matemáticas como algo aislado en su currículum y no siempre entienden que son las mismas cuando se explican en nuestras asignaturas que cuando ellos las emplean en otras (Materiales, Circuitos, ...)? Aquí podríamos debatir sobre notación, formalismo matemático, formas de explicar contenidos de Matemáticas en asignaturas de otras disciplinas y, como hemos comentado anteriormente, hasta de la opinión que de nuestras asignaturas tienen nuestros propios compañeros profesores de otras disciplinas.

Creemos que muchos de nuestros alumnos ven su titulación -y en particular nuestras asignaturas- como una carrera de obstáculos que han de ir superando: asignatura aprobada,... asignatura menos para acabar. Y sin olvidarnos del consabido ¿... y esto para qué sirve?, si yo lo que quiero es ser ingeniero, no matemático.

Porque además, como profesores nosotros podemos saber muchísimas Matemáticas y ser excelentes explicándolas, pero no tener ni idea de que es de lo que de nosotros se necesita tanto para otras asignaturas como para la vida futura del profesional al que estamos formando. Como es bien conocido, la respuesta más tópica que se suele dar en cuanto a la utilidad de las Matemáticas en nuestros estudios, es "... las Matemáticas contribuyen a la formación del alumno", y "... son imprescindibles porque lo capacitan para plantear y resolver problemas". Personalmente, nos negamos a dar estas frases como la única solución aportada en esta charla, aún estando de acuerdo con ambas; evidentemente la Filosofía o la Historia también contribuyen a lo anterior $-\mathrm{y}$ no se estudian en Ingeniería-, y hasta hacer sudokus seguro que ayuda. 
Cada vez somos más de la impresión de que los profesores corremos el riesgo de convertirnos en simples "preparadores de exámenes" (evidentemente, no como lo suelen hacer en las academias particulares a las que suelen asistir muchos de nuestros alumnos): impartimos unas materias y al final, en base a un examen -y poco más- decidimos quien aprueba o quien suspende. Hemos de destacar que en esto no nos diferenciamos tanto de los profesores de bachillerato que se centran única y exclusivamente en preparar las pruebas de acceso -y que afortunadamente nos consta que no son mayoría. Personalmente, cada vez me cuesta más poner un examen -siempre tengo la idea de estar repitiéndome. Pero, ¿acaso no tendemos todos a preguntar casi siempre lo mismo?; y por otro lado -y por decir algo positivo-, ¿no nos damos con un "canto en los dientes" si conseguimos que nuestros alumnos sepan responder a lo que preguntamos?

¿Enseñamos ahora las mismas Matemáticas que hace 30 años? Casi podemos afirmar que sí (posiblemente porque, por ejemplo, el cálculo infinitesimal no ha cambiado tanto, y porque queremos creer que las necesidades que tienen nuestros alumnos $-\mathrm{y}$ titulados- del mismo siguen siendo las mismas). Aunque le podemos ver alguna que otra faceta positiva: aunque sí que podamos impartir contenidos similares, lo que sí afirmamos con rotundidad es que se enseñan las Matemáticas de forma un poco diferente, gracias, entre otras cosas, a las tecnologías puestas a nuestra disposición, y de alguna de las cuales se hablará en estas Jornadas: calculadoras, aula virtual, paquetes matemáticos, gamificación, ...

\section{SOLUCIONES, ... SI LAS HAY}

Tampoco vamos a ser muy originales a la hora de proponer soluciones para la motivación. Solo nos atrevemos a plantear algunas -ya adelantamos que todas ellas conocidas- y esperar al debate posterior para seguro recopilar muchísimas más. Igual de antemano la batalla -para la motivación- la tenemos perdida, ya que la motivación intrínseca, si no se tiene, debería de haber comenzado en edades más tempranas, donde seguro que sí que se podrían emplear muchas de esas técnicas motivacionales que se pueden encontrar en diferentes artículos dirigidos principalmente a profesores en los ciclos anteriores, y alguno de los cuales aparecen referenciados en la bibliografía que acompaña a esta charla. Esta es una queja reiterativa por parte del profesorado: ya que siempre nos quejamos -igual con alguna razón- sobre los conocimientos previos que traen los alumnos cuando llegan a nosotros, también podemos lamentarlos de la falta de motivación que una gran mayoría de alumnos siempre han tenido sobre nuestras asignaturas de Matemáticas.

A continuación nos atrevemos a indicar algunas actividades que consideramos que los profesores podemos llevar fácilmente a la práctica para motivar aunque pueda parecer que ya es tarde- a los alumnos que cursan nuestras asignaturas de Matemáticas, y que seguro pueden extrapolarse sin demasiados problemas a cualquier otra asignatura. Además, creemos que contamos con la ventaja -que igual no siempre existe en los ciclos educativos anteriores- que el alumno es más maduro y que supuestamente sabe lo que 
quiere, aunque no sea otra cosa que aprobar la asignatura (motivación extrínseca); es decir, ya que el alumno suele ser "egoísta" -entendiendo este término, como hemos comentado anteriormente, en sentido positivo; su principal objetivo puede ser conseguir el aprobado más que aprender muchas Matemáticas-, utilicemos este egoísmo en nuestro favor, e igual, añadiendo alguna de las siguientes técnicas, se consigue que la motivación extrínseca se convierta en intrínseca; es decir, que podamos comenzar, como anteriormente se ha indicado, con la motivación extrínseca de querer obtener un aprobado, para acabar motivando intrínsecamente a los alumnos a través de:

- Introducir los temas (conceptos y principales resultados) a través de problemas extraídos de la vida real y de la -supuesta- futura vida laboral que va a desarrollar. Poner ejemplos de donde, por qué y para qué puede utilizar, por ejemplo un ingeniero, la inversa de una matriz. Siempre que sea posible se le intentarán plantear problemas reales, aunque -como mal menor-también podemos plantearles problemas de otras asignaturas donde se utilicen algunos de los contenidos que les enseñamos. Igual lo ideal es hacer una mezcla de ambas cosas y preocuparnos tanto en buscar aplicaciones reales de las Matemáticas a, por ejemplo, la industria como a otras asignaturas de su título -con problemas específicos relacionados con el algebra lineal, cálculo infinitesimal e integral, análisis complejo, ...

- Proponer y dirigir TFG's, actividad que está muy relacionada con la de los problemas de la vida real. Hemos podido constatar que el alumno suele estar mucho más motivado -intrínsecamente- cuando acude a nosotros porque necesita resolver cuestiones matemáticas relacionadas con su TFG. Por ello, y aunque a veces parece que las asignaturas de las áreas básicas no "pintamos" mucho a la hora de dirigir un TFG, ni que tendríamos mucho éxito a la hora de reclutar alumnos para su realización, si somos capaces de relacionar nuestras asignaturas con temas específicos de su título, seguro que el alumno estará mucho más motivado para comprender y utilizar las Matemáticas que les hemos explicado. El problema que ocurre con esta actividad es que el alumno realiza el TFG al final de su etapa educativa, mientras que las asignaturas de Matemáticas suelen cursarse en los primeros cursos, por lo que puede parecer que esta motivación -intrínseca- llega tarde.

- "Bucear" entre el resto de asignaturas de la titulación, para ver que se necesita de nuestras asignaturas en las mismas. Intercambiar experiencias con el resto del profesorado de la titulación, incluyendo a nuestros compañeros que imparten otras asignaturas de Matemáticas. En definitiva, la existencia de una efectiva coordinación horizontal y vertical entre todas las asignaturas del título.

- Preparar manuales para el alumno y otro tipo de material -vídeos, presentaciones, etc. - que hagan que las asignaturas sean más amenas -0 , en su defecto, menos aburridas.

- Rotar con otros compañeros en nuestras asignaturas, ya que a veces, el profesorado suele "acomodarse" cuando imparte muchos cursos seguidos una misma materia, lo que nos puede llevar a cometer siempre los mismos fallos y no ser capaces de conseguir una efectiva motivación de los alumnos. 
- Usar ampliamente todo tipo de tecnologías existentes y que están a nuestra disposición: uso de programas matemáticos, uso de calculadoras... y que eviten el tener que realizar cálculos engorrosos.

- Insistir en la necesaria formación del profesorado, con la asistencia a jornadas, congresos,... donde intercambiar experiencias con otros profesores.

- Utopía a realizar: La existencia de una especialización en docencia de lo que se podría llamar Ingeniería Matemática, es decir, de una rama de las Matemáticas vinculada específicamente a la Ingeniería, y que mostrase a los docentes la enseñanza de las Matemáticas desde una perspectiva propia de la Ingeniería, aportándoles los instrumentos intelectuales y cognitivos necesarios que contribuyan a que las Matemáticas cumplan de la mejor manera posible con las funciones que le han sido asignadas como área especializada del conocimiento humano (Camarena, 2010).

- Y sobre todo, preguntemos a nuestros alumnos su parecer, y no solo nos fiemos en la nota final con la que nos califican en las consabidas encuestas anuales. Ellos, mejor que nadie, y como empleadores últimos de los productos que nosotros les ofrecemos, nos indicarán nuestras fortalezas y debilidades a la hora de conseguir su motivación en nuestras asignaturas.

\section{CONCLUSIONES}

En la presente charla se resalta la importancia que debe tener la motivación en el proceso enseñanza-aprendizaje de las asignaturas de Matemáticas en el caso de los estudios de Ingeniería, aunque fácilmente puede aplicarse a cualquier otra disciplina. Entendemos que la motivación -en estas asignaturases un factor fundamental, no solamente para que el alumno supere las mismas, sino para que descubra por sí mismo su interés en estas materias a raíz, principalmente, de encontrar su utilidad como apoyo esencial tanto para el resto de asignaturas de su titulación, como a la hora de resolver problemas con los que se puede encontrar en su futura vida profesional.

Aunque esta motivación es más evidente cuando los alumnos tienen que afrontar la realización de su TFG, en la exposición anterior aparecen recogidas determinadas técnicas -que posiblemente la mayoría de los docentes aplican en sus respectivas disciplinas- para llevar al alumno desde una motivación inicial extrínseca -aprobar las asignaturas de Matemáticas al ser necesarias para obtener su Grado- a una motivación final intrínseca -una vez que puede comprender lo necesarias que pueden ser estas asignaturas, así como las herramientas que le aportan.

A pesar de la poca motivación que los alumnos suelen traer en nuestra disciplina cuando acceden a la universidad, consideramos que no es tarde para motivarles en Matemáticas, así como para imbuirles en el espíritu del estudio continuo, lo que evidentemente contribuirá a su formación y a su interés por continuar aprendiendo. 


\section{REFERENCIAS}

Camarena, P. (2010). Aportaciones de investigación al aprendizaje y enseñanza de la matemática e ingeniería. Méjico:

http://www.ai.org.mx/ai/archivos/ingresos/camarenagallardo/dra. patricia camarena $\mathrm{g}$ allardo.pdf.

Camarena, P. (2012). La modelación matemática en la formación del ingeniero. Brasil: R. B. E. C. T., vol 5, núm. 3, https://periodicos.utfpr.edu.br/rbect/article/view/1386/902.

García Retana, J. A. (2013). La problemática de la enseñanza y aprendizaje del cálculo para ingeniería. Costa Rica: Revista Educación 37 (1), 29-42.

Gilbert, I. (2005). Motivar para aprender en el aula. Las siete claves de la motivación escolar. Barcelona: Paidós Ibérica.

Gómez Chacón, I. (2005). Motivar a los alumnos de secundaria para hacer Matemáticas. Matemáticas: PISA en la práctica (Curso de formación de profesores). Madrid: Ministerio de Educación y Ciencia.

Maseda, M. (2011). Estudio bibliográfico de la motivación en el aprendizaje de las Matemáticas y propuestas de talleres aplicados en la vida real. Universidad Internacional de La Rioja.

Trejo, E. y otros. (2013). Las Matemáticas en la formación de un ingeniero: la matemática en el contexto como propuesta metodológica. Méjico: Revista de docencia universitaria, vol. 11. 397-424.

Skemp, R. (1980). Psicología del aprendizaje de las Matemáticas. Madrid: Ed. Morata. 